\title{
IDOSOS ON-LINE: TECNOLOGIA COMO RECURSO PARA A APRENDIZAGEM AO LONGO DA VIDA
}

\author{
Meire Cachioni ${ }^{1}$ \\ Karina de Lima Flauzino² \\ Samila Sathler Tavares Batistoni \\ Lilian Ourém Batista Vieira Cliquet $^{4}$ \\ Isabela Zaine ${ }^{5}$ \\ Maria da Graça Campos Pimentel ${ }^{6}$
}

\footnotetext{
1 Psicóloga. Doutora em Gerontologia pelo Programa de Pós-Graduação em Gerontologia UNICAMP. Professora Associada da Escola de Artes, Ciências e Humanidades da Universidade de São Paulo, São Paulo, Brasil. E-mail: meirec@usp.br.

2 Gerontóloga. Mestranda no Programa de Pós-Graduação em Gerontologia. Escola de Artes, Ciências e Humanidades, Universidade de São Paulo, São Paulo, Brasil. e-mail: karinaflauzino@usp.br

3 Psicóloga. Doutora em Gerontologia pelo Programa de Pós-Graduação em Gerontologia UNICAMP. Professora doutora da Escola de Artes, Ciências e Humanidades da Universidade de São Paulo, São Paulo, Brasil. E-mail: samilabatistoni@usp.br.

4 Fonoaudióloga. Mestre em Ciências (Gerontologia) pelo Programa de Pós-Graduação em Gerontologia. Escola de Artes, Ciências e Humanidades, Universidade de São Paulo, São Paulo, Brasil. E-mail: lilian.cliquet@gmail.com.

5 Psicóloga. Doutora pelo Programa de Pós-Graduação em Psicologia da Universidade Federal de São Carlos, São Paulo, Brasil. E-mail: isabela.zaine@gmail.com.

6 Bacharel em Ciências da Computação. Doutora em PhD in Computer Science pela University of Kent at Canterbury, Grã-Bretanha. Titular do Instituto de Ciências Matemáticas e de Computação, Universidade de São Paulo, São Paulo, Brasil. E-mail: mgp@icmc.usp.br.
} 


\begin{abstract}
resumo
As tecnologias digitais de informação e comunicação geram benefícios na vida pessoal e coletiva. Para as pessoas idosas, os recursos tecnológicos promovem saúde, conforto nas atividades diárias, bem-estar social e engajamento no próprio percurso de aprendizagem. No entanto, evidenciam-se dificuldades na utilização de dispositivos móveis pelos idosos, bem como na análise crítica das informações disponibilizadas. Observam-se poucas pesquisas direcionadas ao processo de ensino-aprendizagem no contexto de alfabetização e letramento digital de idosos. Este trabalho apresenta uma intervenção educativa sobre dispositivos móveis para idosos, utilizando-se da tecnologia como forma de apoio personalizado e remoto à aprendizagem de práticas com smartphones e tablets. Um sistema computacional de multiplataforma capaz de coletar e armazenar dados, cadastrar intervenções programadas e executá-las de maneira interativa por meio de um aplicativo foi adotado como recurso pedagógico. No total de 155 idosos participantes, houve um aumento significativo na frequência de uso de $79 \%$ dos recursos do smartphone (Wilcoxon, $\mathrm{p}<0,05)$ no pré e pós-teste. Houve diferenças quanto à autoeficácia geral e alcance de metas via smartphone $(0,18<r>0,66)$, e ainda, quanto à escolaridade e à idade na utilização de recursos do smartphone (de 58\% para $16 \%$ e de $47 \%$ para $21 \%$, respectivamente) no pré e pós-teste. Os resultados preliminares da intervenção apontam possibilidades de desenvolvimento de um modelo educativo de letramento digital para a pessoa idosa.
\end{abstract}

palavras-chave

Tecnologia. Aprendizagem ao longo da vida. Educação. Idosos.

As inovações tecnológicas, interfaces e dispositivos digitais estão cada vez mais presentes nas atividades cotidianas, e, ao mesmo tempo, apresentam-se de forma dinâmica e em constante processo de transformação na sociedade contemporânea. Os recursos digitais estão para além dos postos de trabalho, incluindo-se nas residências e no atendimento de diversos setores e instituições, promovendo uma imersão digital que exige $e$-skills e competências plurais que se associam à globalização e à aprendizagem ao longo da vida (WILLIS, 2006; GIL, 2015). 
As Tecnologias de Informação e Comunicação (TIC) são capazes de transformar a realidade econômica e social das comunidades, tendo o potencial de mediar as aprendizagens nas atividades diárias, potencializando a participação ativa dos cidadãos na sociedade do conhecimento. Espera-se que as pessoas tenham a habilidade de procurar por informações e analisá-las criticamente para resolverem problemas que afetam sua qualidade de vida (BUILDING..., 2019).

As novas tecnologias devem promover tanto a evolução coletiva, como também a valorização da liberdade individual, superando a tensão entre a tradição e a modernidade no âmbito da aprendizagem ao longo da vida. Ou seja, a adaptação e a atualização são essenciais diante das rápidas mudanças presentes na sociedade contemporânea, porém, o percurso de aprendizagem deve-se adequar às necessidades e aos interesses individuais (UNESCO, 2010). Nesta perspectiva, destacam-se as contribuições do campo multidisciplinar da Gerontecnologia, o qual aproxima as áreas do envelhecimento e da tecnologia com intuito de desenvolver técnicas e práticas que ofertam ambientes favoráveis à independência, ao conforto, à saúde e à segurança das pessoas idosas (BARROS, 2012).

As TICs oferecem caminhos para compensar as dificuldades em relação ao tempo e à distância no contexto educacional, sendo facilmente adaptada para atender qualquer nível de aprendizagem do estudante. A realidade virtual permite interações on-line, por diferentes localizações geográficas, com transmissões de áudio, vídeo e textos. Os estudantes com alguma restrição física e/ou sensorial podem se beneficiar com o acesso às informações por meio das plataformas digitais. Desta forma, as experiências tecnológicas abrem possibilidades para a expansão dos programas educativos, considerando o acesso e a participação como dois elementos chaves no cenário da aprendizagem ao longo da vida e novas tecnologias (THORPE, 2000). Este trabalho tem por objetivo apresentar uma iniciativa educativa pioneira do uso de tecnologia como forma de apoio personalizado e remoto à aprendizagem de práticas com smartphones e tablets por idosos.

A iniciativa educativa pretende oportunizar a atuação dos idosos frente ao conhecimento adquirido e estimulá-los ao engajamento no processo de aprendizagem quando estão fora do contexto institucional de ensino. A interação com o dispositivo digital de forma regular e diária pretende elevar os resultados da aprendizagem, em relação à aquisição de conhecimento e habilidades digitais, utilizando-se de um novo recurso tecnológico que favorece o letramento digital. 


\subsection{As tecnologias para as pessoas idosas}

Observam-se dois grandes fenômenos no desenvolvimento das sociedades em todo o mundo. A primeira delas é o envelhecimento populacional, uma megatendência demográfica que gera implicações importantes no desenvolvimento social, econômico e ambiental das comunidades (UN, 2019). A segunda consiste na revolução tecnológica, com o advento das TICs, apresentando-se de maneira dinâmica e transformadora nos processos de desenvolvimento. Observa-se que as interfaces tecnológicas se tornam obsoletas em um período curto de tempo, bem como as habilidades e competências antes exigidas para a sua utilização. A combinação de tais fenômenos geram preocupações quanto às oportunidades tecnológicas voltadas às pessoas idosas (PÁSCOA; GIL, 2015), as quais são identificadas como "imigrantes digitais" ou "nômades digitais", mesmo àquelas que apresentam interação ou exposição às tecnologias em diversas atividades praticadas ao longo da vida (WANG; SIGERSON; CHENG, 2019).

A geração atual de idosos compõe o grupo populacional com menor aproximação aos recursos tecnológicos, seja por sua adoção, utilização ou pela compreensão dos conhecimentos disponibilizados pelas ferramentas digitais (CZAJA; LEE, 2007; HAUK; HÜFFMEIER; KRUMM, 2018). Apesar das pessoas idosas apresentarem atitudes mais positivas em relação ao interesse em adotar e utilizar computadores, tablets e smartphones no cotidiano (HAUK; HÜFFMEIER; KRUMM, 2018), elas possuem um menor senso de autoeficácia em comparação com os indivíduos mais jovens (TSAI; SHILLAIR; COTTEN, 2017). Sugere-se que os sentimentos de frustração frente às rápidas mudanças das tecnologias podem comprometer a apropriação tecnológica pelos idosos, que acabam desistindo do processo de inclusão digital (PÁSCOA; GIL, 2015). A ausência de habilidades digitais e de manejo sobre a veracidade das informações pode afetar tanto a eficiência na utilização da internet, como também impactar diretamente a qualidade de vida das pessoas idosas (WILLIS, 2006). Para Gil (2015), a infoexclusão dos idosos compromete o acesso à informação e o exercício à cidadania plena. Observam-se escassos investimentos em oportunidades de aprendizagem on-line e treinamento sobre o uso das tecnologias para os idosos, bem como os estudos dedicados ao impacto da utilização das tecnologias pelos idosos.

Em termos de práticas pedagógicas, somente a oferta de um novo modelo de aprendizagem não garante a participação dos idosos no meio digital. Deve-se atentar ao planejamento, organização, recursos didáticos e metodológicos adotados nas propostas educativas, adequando-as para atender as especificidades do público idoso (CACHIONI; NERI, 2004). A teoria aliada à prática é fundamental 
para a aprendizagem inicial do uso de tecnologias por idosos, partindo dos conceitos principais sobre o tema para a utilização dos dispositivos móveis e aplicabilidade dos conteúdos no cotidiano (DOLL; MACHADO; CACHIONI, 2016). Tais medidas colaboram para o maior engajamento e persistência dos idosos na adoção e utilização das tecnologias, sendo os principais problemas enfrentados pelos idosos no uso de dispositivos móveis (ZANDBERGEN, 2015). Contribuem, ainda, na prevenção da manifestação de "ansiedade tecnológica" e "ansiedade ao computador", descritas por Fisk et al. (2009) como possíveis reações cognitivas-emocionais de desconfortos dos idosos diante das tecnologias.

Laal (2011) apresenta outra questão importante a considerar: apenas o acesso à informação tecnológica - por meio de computadores, tablets e smartphones - não assegura a inclusão digital das pessoas. A ausência de habilidades necessárias para a utilização da internet entre os idosos é um fator preocupante para a eficiência da inclusão digital (WILLIS, 2006). Destaca-se que o alfabetismo digital é considerado uma etapa inicial para a inclusão digital, abrangendo o acesso e a utilização dos dispositivos e recursos tecnológicos. A compreensão das informações, envolvendo o processo de assimilação, análise crítica e a possibilidade de reelaborar e gerenciar o conhecimento adquirido constitui como a etapa seguinte, definida como letramento digital (MACHADO; LONGHI; BEHAR, 2013).

Por outro lado, a utilização de aplicativos digitais em dispositivos móveis e em computadores proporcionam às pessoas idosas maior conforto ao realizar tarefas cotidianas, acessando portais e serviços on-line que otimizam tempo e investimentos pessoais. As TICs estão entre as tecnologias mais utilizadas pelos idosos, permitindo o fácil acesso às informações em relação à saúde, turismo, mensagens instantâneas, conta bancária, pesquisas, compras, correio eletrônico, entre outros, pelas plataformas de e-government, e-banking, e-shopping, e-health $\mathrm{e}$ e-learning (PÁSCOA; GIL, 2019). As vantagens das inovações tecnológicas são diversas ao constituírem-se como uma importante estratégia para o desenvolvimento pessoal, cognitivo e de bem-estar social (LAAL, 2011; ORDONEZ; YASSUDA; CACHIONI, 2011). No âmbito da saúde, os aplicativos voltados para e-health estão cada vez mais comuns, direcionando serviços e produtos que objetivam atender as demandas de saúde do público idoso.

Há uma tendência das pessoas idosas registrarem atitudes positivas em relação à utilização e à adoção dos computadores pessoais, tablets e smartphones nas tarefas cotidianas (KACHAR, 2010; GATTI; BRIVIO; GALIMBERT, 2017; HAUK; HÜFFMEIER; KRUMM, 2018). Os motivos que levam os idosos a utilizarem aplicativos de redes sociais, por exemplo, envolvem experiências positivas, principalmente relacionadas ao entretenimento e à facilidade de 
comunicação com as pessoas da família e amigos (FERREIRA; TEIXEIRA, 2017). Neste sentido, compreende-se que as tecnologias favorecem além da inclusão digital, também a inclusão social por meio de outras possibilidades de acesso e de desenvolvimento pessoal, afetivo e cognitivo dos usuários (PÁSCOA; GIL, 2015; GIL, 2015). Parece haver um paradoxo das tecnologias em relação aos idosos; por um lado, um crescente acesso deste grupo etário às inovações tecnológicas e, por outro, um aumento da exclusão digital que intensifica a disparidade de conhecimentos entre as gerações (PÁSCOA; GIL, 2019). Neste sentido, propõe-se a reflexão de que a oferta de programas de letramento digital para a geração atual de idosos deve acompanhar o avanço das propostas de acesso e distribuição das informações digitais, na perspectiva da aprendizagem ao longo da vida.

\subsection{A tecnologia como recurso \\ para a aprendizagem ao longo da vida}

A aprendizagem ao longo da vida - lifelong learning - caracteriza-se pela flexibilidade em sua estrutura, incluindo os domínios da educação formal, não formal e informal, podendo ocorrer em qualquer tempo, lugar e em todas as fases do ciclo vital, do início ao final da vida. Considera-se que a aprendizagem ao longo da vida está para além da educação como instrução e treinamento, e sim, presente nas relações familiares, comunitárias, de trabalho, nos meios de informação, no sistema político e na participação social. Portanto, consiste em uma interação das experiências de aprender a conviver, aprender a aprender, aprender a fazer e aprender a ser, dentro e fora das instituições de ensino (DELORS et al., 1996). Neste contexto, a inclusão digital incentiva à permanência ativa e engajada dos idosos na sociedade, contribuindo como antídoto para a solidão, isolamento e para as diferentes maneiras de aprendizado (PÁSCOA; GIL, 2015; HANSEN et al., 2019). Porém, o discurso da aprendizagem ao longo da vida pouco tem sido incorporado nas concepções centrais da educação tecnológica, apesar das novas oportunidades de integração entre as duas temáticas serem criadas a todo o momento (LAAL, 2011).

De acordo com Mansell e Tremblay (2013), o progresso para a sociedade do conhecimento está no desenvolvimento de diferentes recursos para a aprendizagem, favorecendo tanto o acesso como também novos hábitos de apropriação das informações pelas pessoas. Cabe destacar a diferença entre os conceitos de informação e conhecimento, visto que este último requer a interpretação e assimilação das pessoas. $\mathrm{O}$ investimento em aprendizagens como conquista 
do potencial humano, conforme a proposta de educação humanística de Delors et al. (1996) para o século XXI, consiste na aquisição e atualização de variadas habilidades, competências, interesses, conhecimentos e qualificações que permitam às pessoas o atendimento de suas necessidades pessoais e coletivas no contexto social em que se insere. A aprendizagem deve estar ao mesmo tempo, voltada ao aprender "o que" e ao aprender "a ser", alinhada à proposta da sociedade do conhecimento. Desta maneira, a educação centrada no aluno e controlada por ele promove redes de aprendizagem que se estendem ao longo da vida. A inclusão das TICs na construção da rede de aprendizagem auxilia o aluno a torná-lo mais eficiente em seu próprio processo educativo, utilizando-se de softwares e aplicativos com o intuito de melhorar a qualidade de ensino, as pesquisas por informações e aprendizagem (LAAL, 2011).

As experiências educativas promovidas ou compatíveis com a tecnologia digital, compreendidas como tecnologia e-learning, podem ser aplicadas em muitos contextos educativos para idosos (WILLIS, 2006). A educação à distância incluindo recursos de áudio, vídeo e suporte de um tutor on-line oportuniza a aprendizagem para além dos estudos com base no computador, contribuindo para uma atuação digital integrada às outras aplicações, como a televisão e o celular. A realidade virtual promove também uma vasta experiência, permite a participação e interações on-line em tempo real, por pessoas de diferentes regiões geográficas, utilizando-se de transmissão de áudio, vídeo e textos em tela (THORPE, 2000). A tecnologia contribui para o aumento das experiências da aprendizagem virtual e, até mesmo, pode substituir o modelo tradicional da educação presencial (HANSEN et al., 2019). Porém, as pessoas idosas parecem se beneficiar ainda mais com a adoção de uma sincronização mista das propostas educativas - virtual e presencial - as quais propõem um ambiente flexível e contínuo de aprendizagem. Tais iniciativas podem transpor a dificuldade inicial dos alunos idosos em relação à integração tecnológica no ambiente virtual (WILLIS, 2006). Os diferentes tipos de TICs aplicados à aprendizagem mista - on-line learning, internet-based learning, web-based learning e e-learning - propõem recursos semelhantes entre si, utilizando a tecnologia não apenas como uma ferramenta, mas como um recurso pedagógico e de aprendizagem. As questões referentes ao processo de ensino-aprendizagem na utilização e aplicabilidade de dispositivos móveis são temas incipientes por pesquisas científicas na área de educação. 


\section{O Programa "Idosos On-line" \\ fundamentos teóricos e objetivos}

O programa "Idosos On-line" direcionado ao público com 60 anos ou mais é uma das atividades de extensão universitária oferecidas na USP Aberta à Terceira Idade (UATI) na Escola de Artes, Ciências e Humanidades da Universidade de São Paulo (EACH/USP). A coordenação e gerenciamento do referido programa é realizado pelo laboratório de ensino, pesquisa e extensão LabEDUCA60+, vinculado à universidade. O laboratório está registrado no Diretório de Grupos de Pesquisa do CNPq e baseia suas ações na proposta educativa ao longo de toda a vida, envolvendo todos os seus domínios, centrando-se nas potencialidades e necessidades pessoais e coletivas. A criação do programa na UATI surgiu da iniciativa de um discente de graduação do curso de Gerontologia da EACH/USP (ORDONEZ; YASSUDA; CACHIONI, 2011), com objetivos de ensinar as habilidades básicas no uso do computador e da internet (ORLANDI et al., 2015). Com o avanço do programa, as atividades passaram a oportunizar aprendizagens de competências relativas ao uso de tablets e smartphones (ALVARENGA, 2018). Atualmente, o programa está em fase de validação empírica de seus métodos e de pesquisa sobre os fatores de eficácia como intervenção em letramento digital para idosos.

O programa "Idosos On-line" fundamenta-se teoricamente nas premissas de documentos norteadores da educação, os quais englobam os termos de "lifelong", "lifewide" e "life-deep learning" (FAURE et al., 1973; DELORS et al., 1996; BÉLANGER, 2016). As contribuições das áreas da Gerontologia, da Psicologia do Desenvolvimento, de Teorias da Aprendizagem e do Sistema de Informação e Computação são integradas ao programa. Assim, considera-se a heterogeneidade do processo de envelhecimento e as tendências desenvolvimentais associadas à geratividade, sabedoria, integridade e autorregulação do self na velhice são reconhecidas no programa; bem como a agência pessoal no gerenciamento de metas e recursos, e o potencial das variáveis socioculturais e econômicas para a participação dos idosos na sociedade. Adotam-se, ainda, conhecimentos de estudos internacionais com objetivos similares.

$\mathrm{Na}$ literatura, estão dispostas variadas definições sobre letramento digital, de acordo com as especificidades técnicas, cognitivas, motoras e socioemocionais necessárias para manejar as tecnologias digitais (MARTIN, 2005; CALVANI et al., 2008; ESHET-ALKALAI, 2012; GEORGE-WALKER; TYLER, 2014). Uma definição conceitual própria sobre letramento digital foi estabelecida para guiar os objetivos do programa "Idosos On-line" no contexto da UATI, compreendendo como o processo de aquisição e desenvolvimento de competências compostas 
por conhecimentos, habilidades e atitudes positivas na utilização das tecnologias de comunicação e informação para o alcance de metas individuais e coletivas de forma autônoma ou colaborativa. Tal definição diferencia-se de outros conceitos por considerar que o alcance de metas individuais e coletivas entre idosos pode variar dependendo da profundidade da aplicação dos conhecimentos, das competências funcionais e cognitivas e motivações, e para o uso que estabelecem para si mesmos ou para seu grupo/comunidade ao qual pertencem (GEORGE-WALKER; TYLER, 2014).

O programa é composto por dois formatos independentes de letramento digital baseado na tecnologia alvo (computador ou dispositivos móveis - tablets e smartphones). O formato "Idosos On-line: computador" possui algumas particularidades características do ambiente educacional e da didática utilizada. A classe é formada por dez aprendizes idosos, apoiados por dez tutores discentes do curso de graduação em Gerontologia, oferecendo acompanhamento da aprendizagem personalizado diante do computador. O formato "Idosos On-line: dispositivos móveis" busca responder aos avanços da tecnologia e às demandas dos idosos na utilização de dispositivos móveis. Por se tratar de dispositivos digitais pessoais, portáteis e intuitivos, as classes são compostas por vinte aprendizes idosos e cinco tutores auxiliares.

Desde 2017, o Instituto de Ciências Matemáticas e da Computação da Universidade de São Paulo (ICMC/USP) da cidade de São Carlos (SP) é parceiro do programa. O ICMC desenvolveu um aplicativo referente à amostragem de experiência e intervenção programada à distância para o apoio da aprendizagem (ZAINE et al., 2016). Este projeto contou com financiamentos de pesquisa da Fundação de Amparo à Pesquisa do Estado de São Paulo (FAPESP) - n.. do processo: 2017/19915-0. O aplicativo desenvolvido pela ICMC/USP foi incorporado na prática didática das atividades do programa no formato de dispositivos móveis desde 2018, visando ampliar o poder de generalização da aprendizagem, a transferência do conhecimento em contextos para além da sala de aula e verificar, remotamente, o engajamento do aprendiz durante as semanas com a proposição de exercícios referentes ao conteúdo das aulas.

\subsection{ESPIM: a aplicação tecnológica como recurso pedagógico}

O programa no formato "Idosos On-line: dispositivos móveis" utiliza o sistema computacional Experience Sampling and Programmed Intervention Method (ESPIM) como um recurso pedagógico para a alfabetização e letramento digital. 
Desenvolvida pelo ICMC/USP, a multiplataforma ESPIM é uma combinação do Método de Amostragem de Experiência (ESM), do campo da psicologia, e da Abordagem de Sistema Personalizado de Instrução (PSI) da análise de comportamento. A ESM operacionaliza a coleta de informações sistêmicas das experiências das pessoas em ambiente natural e a PSI tem por objetivo a criação de instruções personalizadas para o ensino. Neste sentido, a ESPIM apoia-se no campo da psicologia e na Computação Ubíqua, a qual compreende que as pessoas se utilizam da tecnologia de maneira natural, sendo parte inerente de seu cotidiano, permitindo o auxílio na realização das atividades diárias (WEISER, 1991). O sistema ESPIM oferece suporte e apoio ao planejamento, autoria e execução de intervenções educativas à distância, por meio de três componentes de infraestrutura computacional: o aplicativo móvel, usado pelo aprendiz em acompanhamento; o sistema Web, utilizado por pesquisadores e profissionais para o planejamento das coletas de informações e cadastrar as intervenções (instruções, tarefas, entre outros); e a plataforma Web Service, responsável pelo armazenamento das informações (CUNHA et al., 2018; VIEL et al., 2017).

Os idosos aprendizes recebem as instruções programadas por meio do aplicativo Smarth ESPIM and Sensor Mobile (SENSEM), o qual transforma tais informações em telas interativas, gerando três tipos básicos de intervenção que podem acompanhar em seu enunciado uma mídia, em formato de foto ou vídeo: 1) questão (aberta, de múltipla escolha ou de múltiplas opções); 2) solicitação de mídia (capturar e enviar uma imagem, um áudio ou vídeo); e 3) aplicação externa (abrir outro aplicativo). Além disso, o sistema possibilita controlar múltiplos fluxos, ou seja, de acordo com a resposta do usuário o aplicativo pode seguir um fluxo diferente, apresentando intervenções personalizadas para cada caso. O aplicativo SENSEM registra o horário, a frequência e o tempo de realização de cada tarefa (intervenção), permitindo a repetição pelo usuário de cada exercício. Os pesquisadores e profissionais acompanham todas as informações registradas, juntamente com as intervenções programadas, pela plataforma Web Service. Desta maneira, é possível verificar se o aprendiz idoso cumpriu a tarefa especificada, suas respostas e um resumo de sua interação com o dispositivo, em termos de tempo e quantidade de repetições de cada uma das tarefas. As instruções programadas objetivam reforçar o conteúdo aprendido em sala de aula, estimulando os aprendizes idosos a utilizarem o dispositivo móvel para além do encontro semanal na universidade. Os critérios de inclusão para os aprendizes idosos alvos da intervenção incluíram ter um dispositivo móvel com sistema operacional Android, com versão 4.4 ou superior, com armazenamento mínimo de 8 GB. 


\section{Metodologia "Idosos On-line: dispositivos móveis"}

As intervenções educativas do programa "Idosos On-line: dispositivos móveis" são organizadas em módulos de aula que acompanham o semestre acadêmico do calendário da UATI, totalizando 15 encontros semanais de $2 \mathrm{~h} /$ aula, conforme o Quadro 1.

Quadro 1 - Conteúdo programático do programa "Idosos On-line: dispositivos móveis"

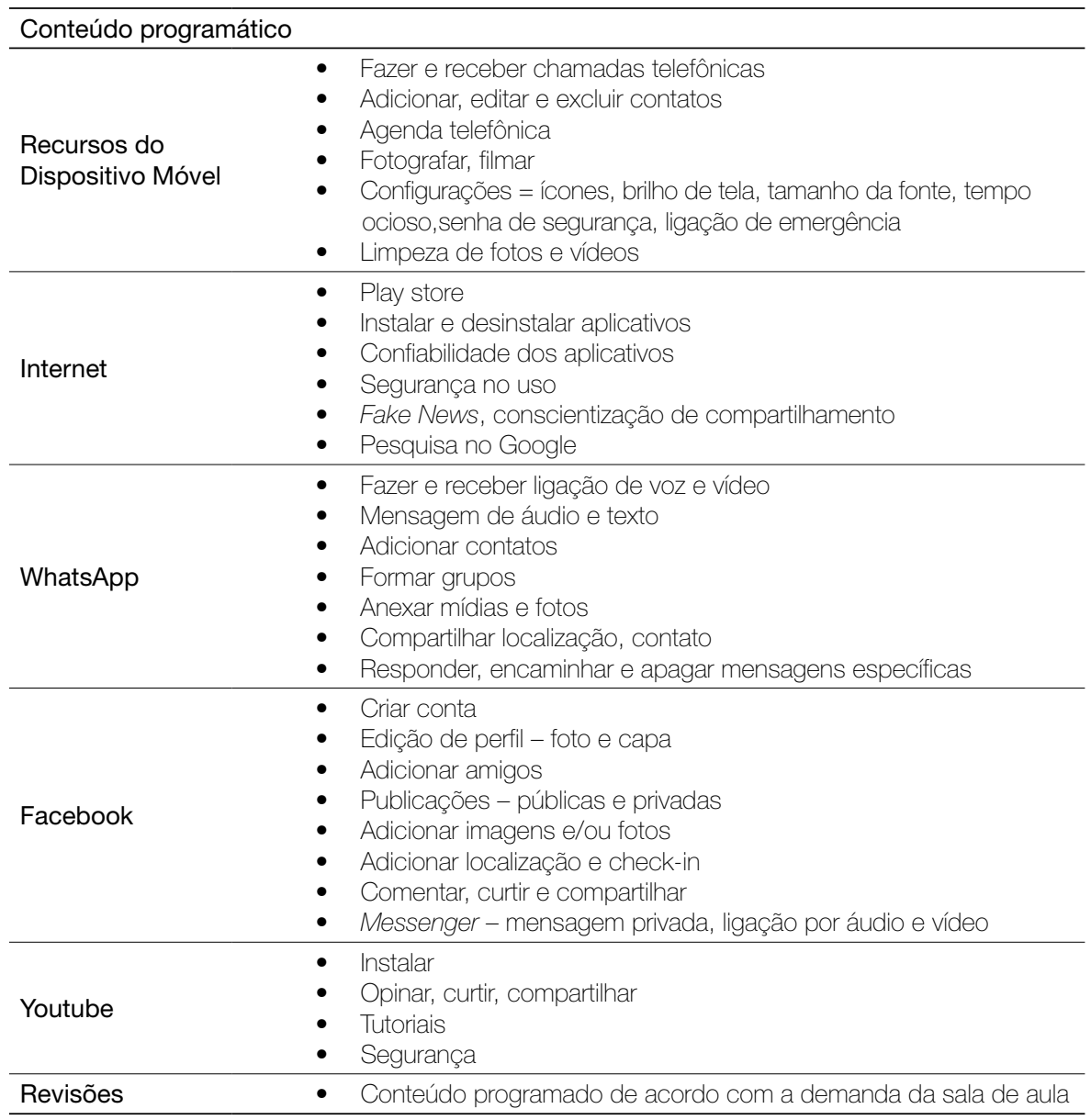

Fonte: Elaborado pelas autoras.

Os encontros são antecedidos e sucedidos por avaliações dos aprendizes idosos para levantamento de dados e identificação de efeitos sobre indicadores 
de bem-estar, cognição e saúde, além de frequência, autoeficácia e desempenho na utilização das tecnologias. No Quadro 2, é possível verificar os instrumentos utilizados no estudo e a descrição de cada um deles.

Quadro 2 - Relação e descrição dos instrumentos utilizados no programa "Idosos On-line: dispositivos móveis"

\begin{tabular}{|c|c|c|c|c|}
\hline \multicolumn{4}{|c|}{ INSTRUMENTOS } & \multirow{2}{*}{$\begin{array}{l}\text { DESCRIÇÃO } \\
\text { Verificou se os dispositivos móveis, } \\
\text { marca, memória, sistema e versão. }\end{array}$} \\
\hline \multirow[b]{2}{*}{ 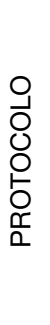 } & \multirow[b]{2}{*}{ 㞾 } & Ficha de Inscrição & $\begin{array}{l}\text { Questionário on-line } \\
\text { ou impresso }\end{array}$ & \\
\hline & & Sociodemográfico & Questionário on-line & $\begin{array}{l}12 \text { questões fechadas e abertas que } \\
\text { compreendem informações sobre: } \\
\text { idade, gênero, escolaridade, estado } \\
\text { civil, profissão, renda familiar, arranjo } \\
\text { familiar. autoavaliação do bem estar } \\
\text { e saúde geral (2p), acesso e uso de } \\
\text { tecnologia (2p) }\end{array}$ \\
\hline \multirow{4}{*}{ 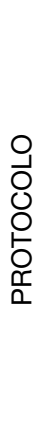 } & \multirow{4}{*}{ 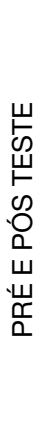 } & TCLE & $\begin{array}{l}\text { Termo de Consentimento } \\
\text { Livre e Esclarecido. Anexo I }\end{array}$ & $\begin{array}{l}\text { Documento impresso em papel } \\
\text { timbrado em } 2 \text { vias, sendo uma para } \\
\text { o aluno e outra para a Instituição }\end{array}$ \\
\hline & & & $\begin{array}{l}\text { MEEM - protocolo on-line } \\
\text { ou impresso - Anexo II }\end{array}$ & $\begin{array}{l}17 \text { questões para resposta orientada } \\
\text { que avaliam o estado cognitivo }\end{array}$ \\
\hline & & Cognitiva & $\begin{array}{l}\text { Fluência Verbal - versão } \\
\text { animais - protocolo } \\
\text { impresso - Anexo III }\end{array}$ & $\begin{array}{l}\text { Evocação de nomes de animais } \\
\text { durante } 1 \text { minuto. }\end{array}$ \\
\hline & & $\begin{array}{l}\text { Conscientização } \\
\text { sobre o } \\
\text { envelhecimento }\end{array}$ & $\begin{array}{l}\text { Questionário on-line } \\
\text { ou impresso }\end{array}$ & $\begin{array}{l}2 \text { questões semiabertas e } 5 \\
\text { questões fechadas. }\end{array}$ \\
\hline \multirow{4}{*}{ 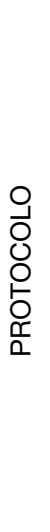 } & \multirow{4}{*}{ 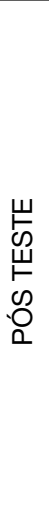 } & $\begin{array}{l}\text { Avaliação das } \\
\text { Tarefas }\end{array}$ & Formulário on-line & $\begin{array}{l}\text { Planilha com a descrição das tarefas } \\
\text { solicitadas para ser completado } \\
\text { com frequência e quantidade da } \\
\text { realização das tarefas. Anexo } 4\end{array}$ \\
\hline & & $\begin{array}{l}\text { Uso do } \\
\text { Dispositivo móvel }\end{array}$ & Questionário on-line & $\begin{array}{l}10 \text { questões fechadas e semiabertas } \\
\text { que buscam informação sobre: } \\
\text { rede móvel, utilização e funções do } \\
\text { dispositivo, facilidades e dificuldades } \\
\text { percebidas quanto ao uso. }\end{array}$ \\
\hline & & $\begin{array}{l}\text { Verificação de } \\
\text { aprendizagem }\end{array}$ & $\begin{array}{l}\text { Formulário impresso ou } \\
\text { on-line - Anexo } 5\end{array}$ & $\begin{array}{l}12 \text { tarefas relacionadas ao conteúdo } \\
\text { programático. }\end{array}$ \\
\hline & & $\begin{array}{l}\text { Diferencial } \\
\text { Semântico do } \\
\text { Aplicativo }\end{array}$ & Documento impresso & $\begin{array}{l}26 \text { itens com } 5 \text { escalas graduadas } \\
\text { entre discordo totalmente e } \\
\text { concordo totalmente. }\end{array}$ \\
\hline
\end{tabular}

Fonte: Elaborado pelas autoras. 
No primeiro dia de aula, os participantes recebem o material de apoio impresso a ser utilizado durante o período do curso e no modo remoto, além de efetuarem a instalação do aplicativo SENSEM nos dispositivos móveis pessoais com o auxílio dos monitores. Todas as instruções de funcionamento e uma simulação prática sobre o aplicativo são executadas em aula, apresentando o aplicativo SENSEM como um "ajudante digital", como um incentivador na interação dos idosos com os dispositivos móveis em períodos fora da sala de aula. Os participantes são informados sobre o recebimento de cinco tarefas por semana remotamente pelo aplicativo, referentes aos conteúdos aprendidos em aula, por exemplo, configuração e manejo do aparelho, de aplicativos, do acesso à internet, redes sociais e uso crítico de dados e informações. Os horários de recebimento das tarefas foram acordados com os alunos, desconsiderando os dias de finais de semana. A execução das tarefas poderia ser realizada de duas maneiras: aguardar a mensagem de alerta diário para iniciar a tarefa, ou autoiniciar a tarefa com o acesso do aplicativo pelo dispositivo móvel.

\section{Resultados}

Já participaram da intervenção educativa 155 idosos, sendo a maioria do sexo feminino $(76,1 \%)$, com média de idade de 68,6 anos de idade $(\mathrm{DP}=6,03)$. As informações obtidas pelos questionários de avaliação pré e pós-intervenção demonstraram um aumento na frequência do uso de dispositivos móveis pelos participantes $(79 \%)$, referente à utilização de 19 recursos do smartphone (Wilcoxon, $\mathrm{p}<0,05$ ), entre eles a comunicação, organização pessoal, gerenciamento de mídia pessoal e aplicativos, navegação na internet e por satélite, e transmissão digital. Houve uma diferença significativa em relação à autoeficácia geral e alcance de metas via smartphone $(0,18<\mathrm{r}>0,66)$. Teste de correlação de Spearman indicou aumento na força de associação entre frequência de utilização e autoeficácia do pré $(\mathrm{r}=0,30 ; \mathrm{p}<0,01)$ para o pós-teste $(\mathrm{r}=0,69 ; \mathrm{p}<0,01)$. Observou-se uma diminuição de $58 \%$ no pré-teste para $16 \%$ no pós-teste em relação à escolaridade e frequência na utilização dos recursos de smartphone, bem como em relação à idade (de $47 \%$ no pré-teste para $21 \%$ no pós-teste).

\section{Discussão e conclusão}

A iniciativa "Idosos On-line: dispositivos móveis" utiliza da tecnologia como um recurso pedagógico personalizável às pessoas idosas participantes de um programa de extensão universitária. Do ponto de vista qualitativo, 
a associação de um aplicativo aos métodos educativos já desenvolvidos pelo grupo tem otimizado os processos e favorecido o engajamento digital dos idosos. Embora a intervenção educativa seja fundamentada em pressupostos teóricos e evidencias de pesquisas correntes, tal experiência ainda carece de investigações mais amplas sobre suas vantagens em relação aos métodos tradicionais em termos de eficiência e eficácia. Os resultados preliminares são promissores e apontam possibilidades de desenvolvimento de um modelo educativo de letramento digital para a pessoa idosa.

\author{
ONLINE ELDERLY: TECHNOLOGY AS A \\ RESOURCE FOR LIFELONG LEARNING
}

\title{
abstract
}

Digital information and communication technologies generate benefits in personal and collective life. For the elderly, technological resources promote health, comfort in daily activities, social well-being and engagement in the learning path itself. However, difficulties are shown in the use of mobile devices by the elderly, as well as in the critical analysis of the information available. There is little research directed to the teaching-learning process in the context of digital literacy of the elderly. This paper presents an educational intervention on mobile devices for the elderly, using technology as a form of personalized and remote support for learning practices with smartphones and tablets. The research adopted a multiplatform computational system capable of collecting and storing data, registering scheduled interventions and executing them interactively through an application. In a total of 155 elderly participants, there was a significant increase in the frequency of use of $79 \%$ of smartphone resources (Wilcoxon, $p<0.05$ ) in the pre- and post-test. There were differences in overall self-efficacy and goal range via smartphone $(0.18<r>0.66)$, and also, regarding schooling and age in the use of smartphone resources (from 58\% to $16 \%$ and from $47 \%$ to $21 \%$, respectively) in the pre and post-test. The preliminary results of the intervention indicate possibilities for the development of an educational model of digital literacy for the elderly.

keywords

Technology. Lifelong Learning. Education. Elderly People 
referências

ALVARENGA, Glaucia Martins de Oliveira et al. Idosos e inclusão digital com tablete: uma revisão sistemática da literatura. Estudos Interdisciplinares sobre o Envelhecimento, Porto Alegre, v. 23, n. 1, p. 125-142, 2018.

BARROS, Elaine Joana Lima et al. Gerontotecnologia educativa voltada ao idoso estomizado à luz da complexidade. Revista Gaúcha de Enfermagem, Porto Alegre, v. 33, n. 2, p. 95-101, 2012.

BÉLANGER, Paul. Self-Construction and social transformation: lifelong, lifewide and life-deep learning. Tradução de Albert Daigen. Germany: UNESCO/UIL, 2016. Disponível em: https://unesdoc.unesco.org/ark:/48223/pf0000244440. Acesso em: 2 nov. 2019.

BUILDING knowledge societies. United Nations Educational, Scientific and Cultural Organization (UNESCO), [s. l.], 2019. Disponível em: https://en.unesco.org/themes/ building-knowledge-societies. Acesso em: 2 out. 2019

CACHIONI, Meire; NERI, Anita Liberalesso. Educação e velhice bem-sucedida no contexto das universidades da terceira idade. In: NERI, Anita; YASSUDA, Mônica Sanches (org.). Velhice Bem-Sucedida: aspectos afetivos e cognitivos. Campinas: Papirus, 2004. p. 29-49.

CALVANI, Antonio et al. Models and instruments for assessing digital competence at school. Journal of e-Learning and Knowledge Society, Reggio Emilia, v. 4, n. 3, p. 183-193, 2008

CUNHA, Bruna Carolina Rodrigues et al. Authoring of interventions with on-demand generation of screen for mobile devices. In: SIMPÓSIO BRASILEIRO DE SISTEMAS MULTIMÍDIA EWEB, 24., 2018, Salvador. Anais [...]. Porto Alegre: SBC, 2018. p. 145-149.

CZAJA, Sara; LEE, Chin Chin. The impact of aging on access to technology. Universal Access in the Information Society, Berlin, v. 5, n. 4, p. 341-349, 2007.

DELORS, Jacques et al. Learning: the treasure within; report to UNESCO of the International Commission on Education for the Twenty-first Century (highlights). Paris: UNESCO, 1996.

DOLL, Johannes; MACHADO, Leticia Rocha; CACHIONI, Meire. O idoso e as novas tecnologias. In: FREITAS, Elizabete Viana de; PY, Ligia (ed.). Tratado de geriatria e gerontologia. 4. ed. Rio de Janeiro: Guanabara Koogan, 2016. p. 3585-3603.

ESHET-ALKALAI, Yoram. Thinking in the digital era: a revised model for digital literacy. Issues in Informing Science and Information Technology, California, v. 9, p. 267-276, 2012

FAURE, Edgar et al. Aprender a Ser: la educación del futuro. Tradução de Carmem Paredes de Castro. 2. ed. Madri: Alianza/UNESCO, 1973.

FERREIRA, Michele Cristina; TEIXEIRA, Karla Maria Damiano. O uso de redes sociais virtuais pelos idosos. Estudos Interdisciplinares sobre o Envelhecimento, Porto Alegre, v. 22, n. 3, p. 153-167, 2017.

FISK, Arthur D. et al. Designing for older adults: principles and creative human factors approaches. 2. ed. Boca Raton: CRC Press, 2009.

GATTI, Fabiana; BRIVIO, Eleonora; GALIMBERT, Carlo. The Future Is Ours Too: a training process to enable the learning perception and increase self-efficacy in the use of tablet in the elderly. Educational Gerontology, Philadelphia, v. 43, n. 4, p. 209-224, 2017.

GEORGE-WALKER, Linda de; TYLER, MarkA. Connected older adults: conceptualising their digital participation. Journal of Literacy and Technology, Boca Raton, v. 15, n. 2 p. 200-2014, 2014 
GIL, Henrique. Educação gerontológica na contemporaneidade: a gerontagogia, as universidades de terceira idade e os nativos digitais. Revista Brasileira de Ciências do Envelhecimento Humano, Passo Fundo, v. 12, n. 3, p. 212-233, set./ dez. 2015. Disponível em: https://repositorio.ipcb.pt/bitstream/10400.11/5382/1/ Educa\%C3\%A7\%C3\%A30\%20gerontol\%C3\%B3gica.pdf. Acesso em: 4 out. 2019.

HANSEN, Robert Jack et al. Enhancing older adult access to lifelong learning institutes through technology-based instruction: a brief report. Gerontology and Geriatrics Education, Philadelphia, v. 41, n. 3, p. 342-351, 2019

HAUK, Nathalie; HÜFFMEIER, Joachim; KRUMM, Stefan. Ready to be a silver surfer? A meta-analysis on the relationship between chronological age and technology acceptance. Computers in Human Behavior, Amsterdam, v. 84, p. 304-319, 2018.

KACHAR, Vitória. Envelhecimento e perspectivas de inclusão digital. Kairós Gerontologia, São Paulo, v. 13, n. 2, 2010. Disponível em: https://revistas.pucsp.br/kairos/ article/view/5371. Acesso em: 6 nov. 2019.

LAAL, Marjan. Impact of technology on lifelong learning. Procedia: Social and Behavioral Sciences, Nicosia, v. 28, p. 439-443, 2011. Disponivel em: https://www.sciencedirect. com/science/article/pii/S1877042811025237. Acesso em: 1 out. 2019.

MACHADO, Lucília Regina; LONGHI, Magali Teresinha; BEHAR, Patricia Alejandra. Domínio Tecnológico: saberes e fazeres na educação a distância. In: BEHAR, Patricia Alejandra (org.). Competências na Educação a Distância. Porto Alegre: Penso, 2013.

MANSELL, Robin; TREMBLAY, Gaëtan. Renewing the knowledge societies vision for peace and sustainable development. Paris: UNESCO, 2013. Disponível em: https://unesdoc.unesco.org/ark:/48223/pfo000224531. Acesso em: 1 out. 2019.

MARTIN, Allan. DigEuLit: a european framework for digital literacy - a progress report. Journal of eLiteracy, Glasgow, v. 2, n. 2, p. 130-136, 2005.

ORDONEZ, Tiado Nascimento; YASSUDA, Mônica Sanches; CACHIONI, Meire. Elderly online: effects of a digital inclusion program in cognitive performance. Archives of Gerontology and Geriatrics, Amsterdam, v. 53, n. 2, p. 216-219, 2011.

ORGANIZAÇÃO DAS NAÇÕES UNIDAS PARA A EDUCAÇÃO, A CIÊNCIA EA CULTURA (UNESCO). Educação: um tesouro a descobrir, relatório para a UNESCO da Comissão Internacional sobre Educação para o Século XXI (destaques). Tradução de Guilherme João de Freitas Teixeira. Brasília: UNESCO, 2010. Disponível em: https://unesdoc. unesco.org/ark:/48223/pf0000109590_por. Acesso em: 1 out. 2019.

ORLANDI, Brunella Della Maggiori et al. Indicadores de validade e confiabilidade do Questionário de Atitudes frente aos Computadores (QUAC) entre idosos brasileiros. Exedra, Coimbra, v. 1, p. 47-60, 2015.

PÁSCOA, Gina; GIL, Henrique. Envelhecimento e tecnologia: desafios do século XXI. In: IBERIAN CONFERENCE ON INFORMATION SYSTEMS AND TECHNOLOGIES, 14. 2019, Coimbra. Anais [...]. Piscataway: IEEE, 2019.

PÁSCOA, Gina; GIL, Henrique. As TIC como antídoto para a solidão e isolamento do cidadão sênior: uma plataforma essencial para alcançar o bem-estar mental e social. Sensos, Portugal, v. 5, n. 1, p. 65-77, 2015.

THORPE, Mary. New Technology and lifelong learning. Washington: ERIC, 2000. Disponível em: https://eric.ed.gov/?id=ED445187. Acesso em: 2 out. 2019.

TSAI, Hsin Yi Sandy; SHILLAIR, Ruth; COTTEN, Sheila. Social Support and "Playing Around": an examination of how older adults acquire digital literacy with tablet computers. Journal of Applied Gerontology, California, v. 36, n. 1, p. 29-55, set. 2017.

UNITED NATIONS (UN). Department of Economic and Social Affairs. World Population Prospects 2019: highlights. New York: UN, 2019. Disponivel em: https://population.un.org/ wpp/Publications/Files/WPP2019_Highlights.pdf. Acesso em: 27 set. 2019. 
VIEL, Caio et al. Personalized Ubiquitous Data Collection and Intervention as Interactive Multimedia Documents. In: SYMPOSIUM ON DOCUMENT ENGINEERING, 17., 2017, Valletta. Anais [...]. New York: ACM, 2017. p. 223-226.

WANG, Hsyn-Yi; SIGERSON, Leif; CHENG, Cecilia. Digital Nativity and Information Technology Addiction: age cohort versus individual difference approaches. Computers in Human Behavior, Amsterdam, v. 90, p. 1-9, 2019.

WEISER, Mark. The Computer for the 21st Century. Scientific American, New York, v. 265, n. 3, p. 223-226, 1991.

WILLIS, Sherry L. Technology and learning in current and future generations of elders. Generations, California, v. 30, n. 2, p. 44-48, 2006. Disponivel em: https://pdfs.semanticscholar.org/1480/8b60bab381ef392a93092f2a3d3e148aec39.pdf. Acesso em: 2 out. 2019.

ZAINE, Isabela et al. An Ubiquitous Data Collection and Programmed Intervention System using ESM and Mobile Devices. In: BRAZILIAN SYMPOSIUM ON MULTIMEDIA AND THE WEB, 22., 2016, Teresina. Anais [...]. New York: ACM, 2016. p. 13-14.

ZANDBERGEN, Ruud. Predicting persistency of usability problems based on error classification: a longitudinal study on improving mobility for the elderly. 2015. 107f. Dissertação (Mestrado em Engenharia) - Faculty of Behavioural, Management and Social sciences, University of Twente, Enschede, 2015

Recebido: 25/04/2020

Aceito: 30/05/2020 